developing a tool for quantifying dactylitis according to the circumference of the affected digit and the degree of tenderness.

The authors tested their new instrument on seven patients who had suffered from psoriatic arthritis for more than 5 years on average. Each patient was assessed twice by each of five examiners, who had varying degrees of expertise in rheumatology. The examination started with identification of the affected digit(s). The digital circumference at the level of the proximal phalanx was then measured using a plastic loop device. This was then expressed as a ratio relative to the contralateral digit. In cases where the contralateral digit was also affected by dactylitis, standard reference tables for digital circumference were used instead. Next, the examiners made a subjective measurement of tenderness by applying moderate pressure to the affected digit, and recording the patient's response on a scale of 0 to 3 . A final score was then generated using the digital-circumference ratio and the tenderness score.

The researchers found that the assessment tool was quick and simple to use, and that interobserver and intraobserver reliability was good. They suggest that this instrument should be validated in a larger group of patients, using further tests of responsiveness and established imaging methods.

Ruth Kirby

Original article Helliwell PS et al. (2005) Development of an assessment tool for dactylitis in patients with psoriatic arthritis. J Rheumatol 32: 1745-1750

\section{Improved outcome in acute or subacute interstitial pneumonia using combination therapy}

Patients with polymyositis or dermatomyositis are at risk of developing acute or subacute interstitial pneumonia (A/SIP), which can be fatal despite high-dose prednisolone therapy.

Kameda and colleagues undertook a pilot trial of combined immunosuppressive therapy (highdose prednisolone, $10-30 \mathrm{mg} / \mathrm{kg}$ intravenous pulse cyclophosphamide every 3-4 weeks and $2-4 \mathrm{mg} / \mathrm{kg} /$ day cyclosporine A) for A/SIP in patients with polymyositis or dermatomyositis. In the groups included in this study, all patients who went on to develop A/SIP had dermatomyositis. Analysis of the outcomes of patients with A/SIP treated with conventional regimens prior to the trial showed that $75 \%$ died despite immunosuppressive therapy (single or sequential). Use of the pilot combined regimen in 10 patients with A/SIP doubled the survival rate to $50 \%$ : 5 patients survived and are doing well 2 years later; 5 patients died of respiratory failure within 3 months. Analysis of clinical findings in those patients who developed AVSIP showed characteristic features of mild myositis, palmar papule, fever and negative or low titer of antinuclear antibodies. Of these, the authors considered palmar papules to be a likely indication of the presence or development of A/SIP and potential fatal outcome.

The immediate use of combined immunosuppressive therapy improved outcome in patients with A/SIP and dermatomyositis, although early and aggressive treatment was still unsuccessful in around half of such patients.

Carol Lovegrove

Original article Kameda $\mathrm{H}$ et al. (2005) Combination therapy with corticosteroids, cyclosporine $\mathrm{A}$, and intravenous pulse cyclophosphamide for acute/subacute interstitial pneumonia in patients with dermatomyositis. J Rheumatol 32: $1719-1726$

\section{Cytokine genotype relates to outcome in juvenile rheumatoid arthritis}

Cytokine gene polymorphisms have been associated with the risk of developing a number of autoimmune diseases, including systemic juvenile idiopathic arthritis. As some singlenucleotide polymorphisms (SNPs) have been associated with variations in gene expression, it is conceivable that such functional polymorphisms might affect inflammatory processes and, potentially, disease outcome. On this premise, Oen and colleagues determined the genotypes of SNPs for a number of proinflammatory (interleukin-6, interferon- $\gamma$, tumor necrosis factor) and anti-inflammatory (interleukin-10, transforming growth factor- $\beta$ ) cytokines in patients with juvenile rheumatoid arthritis (JRA) who had taken part in a long-term outcome study. Cytokine genotypes were then correlated with clinical outcome in univariate and multivariate tests.

Of the cytokine genotypes examined, interleukin-6 genotype 174G/G showed a significant correlation with pain in JRA. As pain is the largest contributor to variation in physical 